\title{
Obstrucción duodenal congénita de etiología múltiple: Reporte de un caso con malrotación intestinal, bandas de Ladd y membrana duodenal intrínseca como causantes de obstrucción intestinal en el neonato
}

Helí de Jesús Rueda Chaparro*, Victoria Cárdenas Ramírez², Liliana Arias Álvarez³.

1. Médico residente de Radiología. Pontificia Universidad Javeriana. Bogotá, Colombia.

2. Médico residente de Pediatría. Pontificia Universidad Javeriana. Bogotá, Colombia.

3. Médico Radiólogo. Departamento de Radiología, Hospital Universitario San Ignacio. Bogotá, Colombia.

Congenital duodenal obstruction of multiple etiology: Case report with intestinal malrotation, Ladd bands and intrinsic duodenal membrane as causes of intestinal obstruction in the neonate

Resumen: La obstrucción duodenal congénita agrupa un amplio espectro de anomalías en el desarrollo del intestino delgado; se clasifica según su etiología tanto en extrínseca como intrínseca. Su presentación es relativamente común, teniendo una incidencia de 1: 2.500-10.000 nacidos vivos y normalmente presenta asociación con aneuploidías (como la trisomía 21) y malformaciones cardíacas. Las imágenes tienen un papel importante en la aproximación diagnóstica y la radiografía de abdomen simple, es el primer escalón en la aproximación de la obstrucción intestinal. El tratamiento involucra una exploración quirúrgica del abdomen con corrección de los defectos encontrados.

Este es un reporte de caso de un neonato que presentó obstrucción duodenal congénita de etiología tanto extrínseca (malrotacion intestinal y bandas de Ladd) como obstrucción intrínseca (membranas duodenales). Palabras claves: Congénito, Malrotación, Obstrucción duodenal.

Abstract: Congenital duodenal obstruction groups together a broad spectrum of abnormalities in the development of the small intestine; it is classified according to its etiology as both extrinsic and intrinsic. Its presentation is relatively common, having an incidence of 1: 2.500-10.000 live births and it is normally associated with aneuploidy (such as trisomy 21) and cardiac malformations. Images play an important role in the diagnostic approach, with simple abdominal radiography being the first step in the approximation of intestinal obstruction. The treatment corresponds to a surgical exploration of the abdomen with resolution of the defects found. This is a case report of a newborn who presented congenital duodenal obstruction of both extrinsic etiology (intestinal malrotation and Ladd bands) and intrinsic obstruction (duodenal membranes). Keywords: Congenital, Duodenal obstruction, Malrotation.

Rueda H., et al. Obstrucción duodenal congénita de etiología múltiple: Reporte de un caso con malrotación intestinal, bandas de Ladd y membrana duodenal intrínseca como causantes de obstrucción intestinal en el neonato. Rev Chil Radiol 2019; 25(4): 146-149.

*Correo electrónico: Heli Rueda / helirueda@hotmail.com

Trabajo enviado el 08 de junio de 2019. Aceptado para publicación el 29 de noviembre de 2019.

\section{Presentación del caso}

Recién nacido a término con diagnóstico perinatal de trisomía 21 libre. Se realiza ecocardiograma posnatal que evidencia canal auriculoventricular completo y comunicación interventricular del tracto de entrada de $12 \mathrm{~mm}$ que se comporta como ventrículo común.

A las 24 horas de vida se inició vía oral con posterior aparición de múltiples episodios eméticos por lo cual se solicita radiografía de abdomen simple con evidencia de patrón gaseoso intestinal anormal (Figura 1). Por este motivo, se solicita radiografía de vías digestivas altas para ampliar diagnóstico la cual muestra imágenes sugestivas de estenosis duodenal (Figura 2). Posteriormente es valorado por el grupo de 


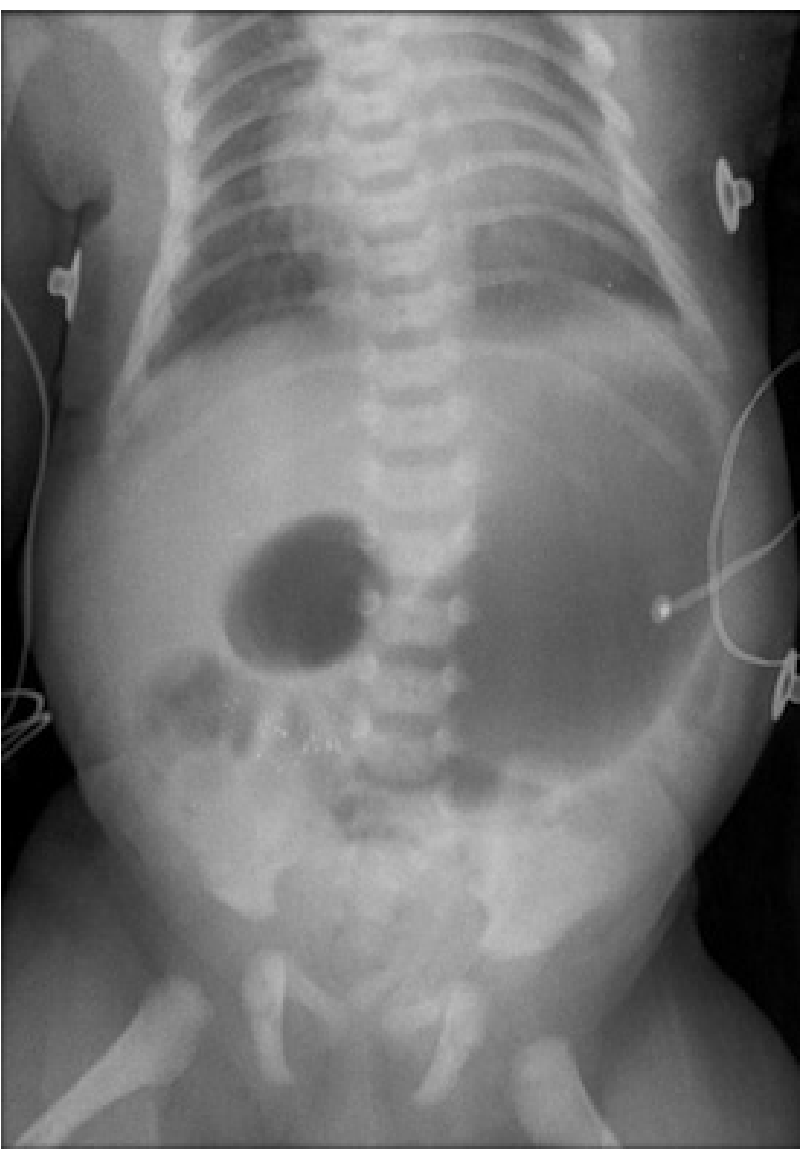

Figura 1: Radiografía de abdomen. Proyección anteroposterior. Se observan dos burbujas localizadas en hipocondrio izquierdo y paravertebral derecha en relación con marcada distensión de la cámara gástrica y del bulbo duodenal, con escaso gas en intestino delgado proximal. No se visualiza gas en recto. Artificios radiopacos puntiformes hacia la fosa ilíaca derecha secundarios al chasis.

cirugía pediátrica, quien considera necesario realizar exploración quirúrgica de urgencias. Se realiza laparotomía, encontrando malrotación intestinal con evidencia intraoperatoria de bandas de Ladd que comprimen el duodeno y cambios del calibre en la segunda porción del duodeno con presencia de membrana duodenal, cuyo tratamiento es corrección de malrotación intestinal, liberación de bandas de Ladd, apendicetomía, resección de membrana duodenal y plastia duodenal sin complicaciones inmediatas.

A los 7 días de posoperatorio se inicia aporte enteral de forma progresiva. Sin embargo, 6 días después, el paciente presenta aumento de perímetro abdominal y deterioro hemodinámico, razón por la cual se le ordena un hemograma que demuestra leucocitosis y neutrofilia; se considera suspender vía oral y se avanza sonda orogástrica con drenaje de líquido bilioso y sanguinolento. Posteriormente, se indica toma de radiografía de abdomen con sospecha de perforación

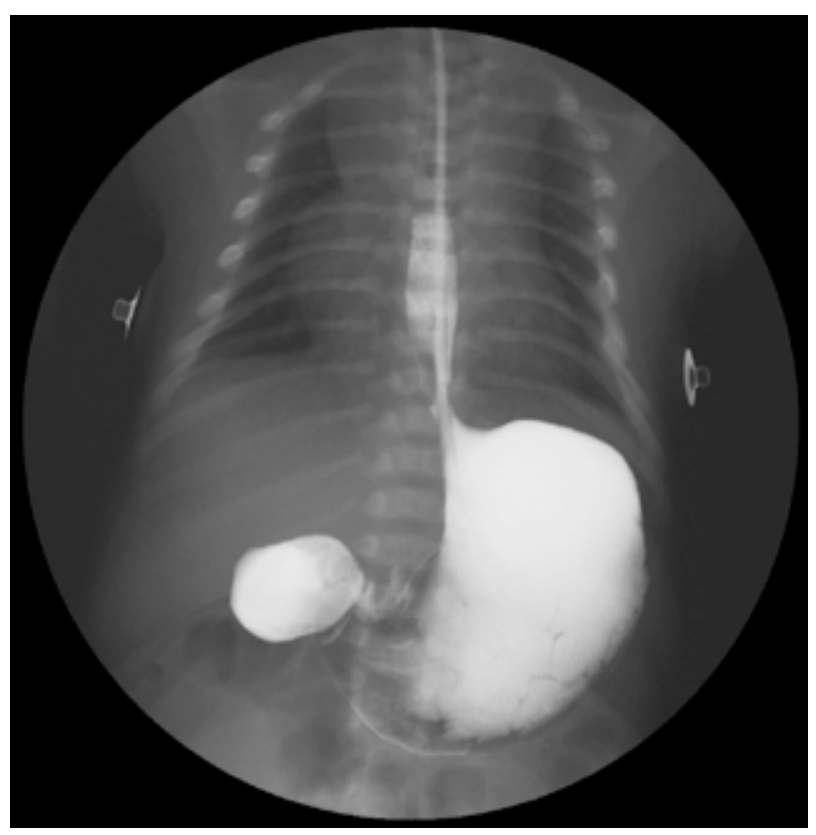

Figura 2: Radiografía de vías digestivas altas, proyección anteroposterior. Opacificación con marcada dilatación de estómago y bulbo duodenal, sin paso del medio de contraste durante el tiempo de fluoroscopia. Se observa escaso gas distal, estos hallazgos sugieren estenosis duodenal.

intestinal (Figura 3a y 3b) debido a choque séptico del paciente. Se inicia cubrimiento antibiótico de amplio espectro y se realiza laparotomía exploratoria de urgencia que evidencia necrosis intestinal masiva con peritonitis generalizada que conllevan al fallecimiento del paciente.

\section{Discusión}

La obstrucción duodenal congénita agrupa un amplio espectro de anomalías en el desarrollo del intestino delgado, cuyo desenlace común concluye en la disminución del calibre de la luz intestinal generando síntomas gastrointestinales obstructivos. Su desarrollo tiene lugar en la vida intrauterina durante el período embrionario, e incluye desde fallas en el desarrollo del intestino, alteraciones en la recanalización intestinal y malrotaciones, hasta compresiones extrínsecas de las estructuras anatómicas cercanas al duodeno ${ }^{(1)}$.

Su presentación es relativamente común, teniendo una incidencia de 1: 2.500-10.000 nacidos vivos y normalmente presenta asociación con aneuploidías (como la trisomía 21) y malformaciones cardíacas ${ }^{(1,2,3)}$.

La obstrucción duodenal congénita puede clasificarse según su etiología en intrínseca y extrínseca. Las intrínsecas agrupan la atresia duodenal, estenosis duodenal y las membranas duodenales. Las extrínsecas incluyen las bandas de Ladd, el páncreas anular, la malrotación intestinal, duplicaciones duodenales, vena porta anterior y volvulaciones ${ }^{(3)}$. La presencia de 

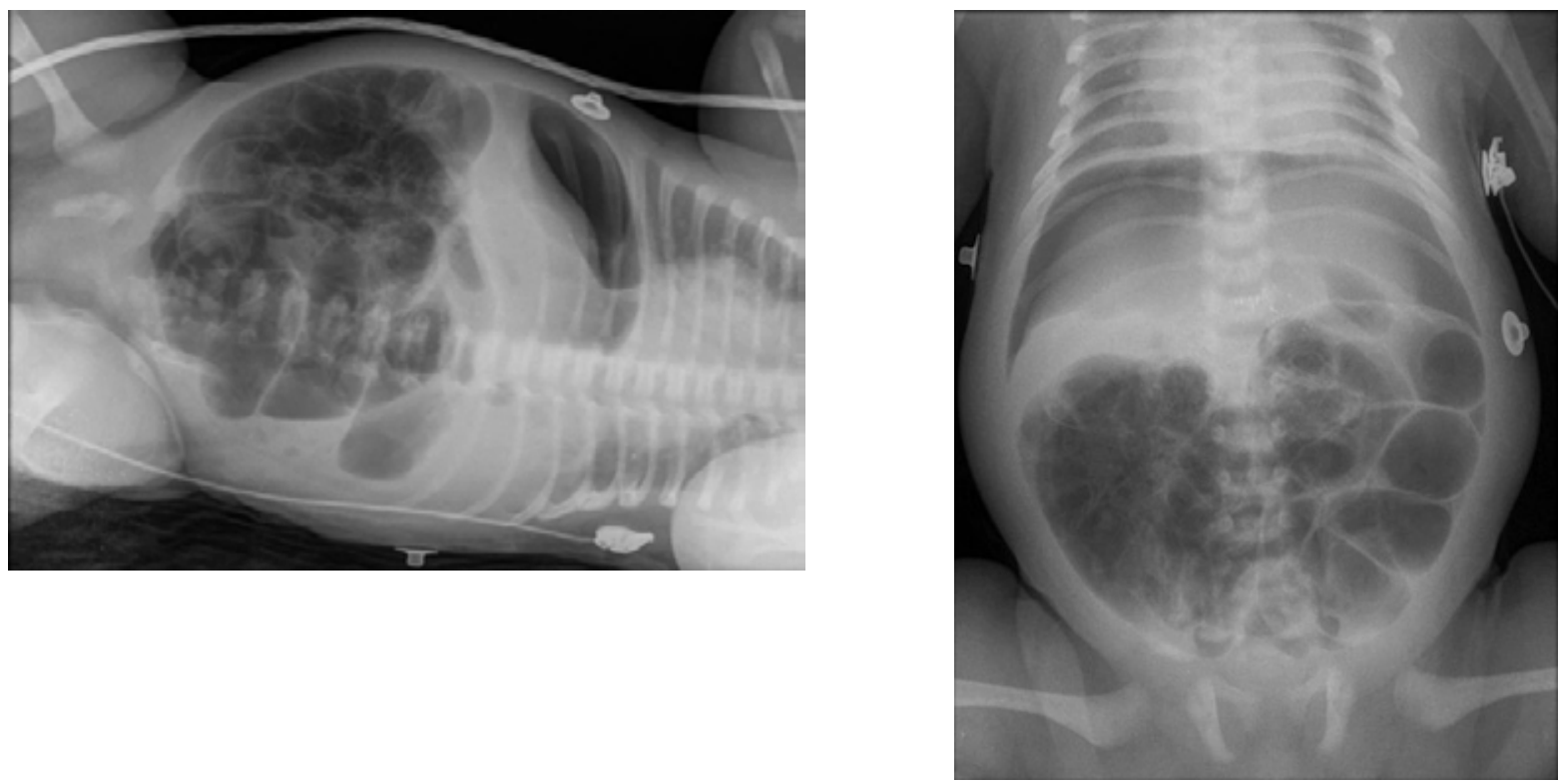

Figura 3a y 3b: Radiografía de abdomen. Proyecciones en decúbito lateral izquierdo y supino posquirúrgicas, en las que se identifica neumoperitoneo y dilatación de asas intestinales delgadas. Cabeza de flecha: Engrosamiento de la pared intestinal de asa visualiazada en mesogastrio con radiolucidez lineal en área declive compatible con neumatosis intestinal.

una etiología única de la obstrucción duodenal congénita es común entre los diagnósticos del neonato, sin embargo, la combinación de causas extrínsecas e intrínsecas en el mismo cuadro clínico es sumamente rara $^{(4)}$. La particularidad de este reporte de caso radica en la presentación de tres etiologías causantes: La malrotación intestinal y bandas de Ladd como extrínsecas y una membrana duodenal en la segunda porción del duodeno como intrínseca.

El desarrollo normal del intestino ocurre a partir de la 4ta a 6ta semana de gestación, en el que el intestino primitivo tiene la disposición de un tubo que a medida que avanza el embarazo (semana 10) aumenta su tamaño y comienza su plegamiento y maduración sobre sí mismo para dar origen a las estructuras anatómicas finales, rotando $270^{\circ}$ sobre su propio eje en relación con la arteria mesentérica superior ${ }^{(5)}$. La rotación normal del intestino debe permitir que la unión duodeno-yeyunal se encuentre en el cuadrante abdominal superior izquierdo y el ciego en el cuadrante abdominal inferior derecho(6). Ambas estructuras se encuentran suspendidas por el mesenterio. Cualquier interrupción en dicha rotación puede conllevar a una alteración en la disposición de las asas intestinales y favorecer su obstrucción. Las bandas de Ladd corresponden a desórdenes en la fijación de dichas estructuras, estas bandas son prolongaciones fibrosas intraperitoneales producto de una fijación anormal del mesenterio en pacientes con malrotación intestinal; se extienden desde el ciego malrotado, pasando a través del duodeno comprimiéndolo hasta llegar a su inserción en el hígado y deben su nombre al Dr. William E. Ladd, pionero en la cirugía pediátrica( ${ }^{(1)}$.

Por último, las membranas duodenales ocurren por una falla en la recanalización del duodeno durante la 5ta semana de gestación, corresponde a una membrana de tejido hecha de mucosa y submucosa intestinal. Su localización más común es en la segunda porción del duodeno(7,8).

La sintomatología cardinal de la obstrucción duodenal congénita es el vómito posprandial, el cual inicialmente es de contenido alimentario y progresa a bilioso. La distensión abdominal puede acompañar dicho síntoma ${ }^{(1,4,8)}$.

Las imágenes diagnósticas juegan un papel importante en la aproximación diagnóstica de esta entidad. La radiografía de abdomen constituye el primer escalón en la aproximación de la obstrucción intestinal en el neonato, donde se observa el signo clásico de la "doble burbuja" por la distensión de estómago y la primera porción del duodeno(1), si no hay evidencia de gas distal, la atresia duodenal debe ser la primera posibilidad diagnóstica, ahora bien, si existe la presencia de escaso gas distal ha de considerarse la estenosis duodenal o membrana duodenal como impresión diagnóstica ${ }^{(8)}$.

Los estudios de fluoroscopia, como la radiografía de vías digestivas altas, confiere una información valiosa para el cirujano en su criterio clínico. Si bien los hallazgos de atresia duodenal como el signo de 
la "doble burbuja" y ausencia de gas distal en la radiografía de abdomen son patognomónicos y no requieren de estudio adicional para definir manejo quirúrgico, la radiografía de vías digestivas altas en el contexto de la presencia de gas distal junto con la "doble burbuja" en la radiografía convencional de abdomen ayuda en el diagnóstico diferencial entre estenosis duodenal y membranas duodenales, de un vólvulo del intestino medio. Éste último es una urgencia quirúrgica. La diferencia radica en que, la estenosis y la membrana duodenal ocurren en la segunda porción del duodeno y el vólvulo a partir de la tercera porción ${ }^{(8)}$.

El uso del ultrasonido como modalidad diagnóstica en el abordaje del vómito en el neonato ha cobrado gran importancia actualmente. Esto se debe a que permite evaluación en tiempo real del abdomen y la ausencia de radiación ionizante. Los hallazgos ecográficos que sugieren malrotación intestinal neonatal comprenden la evaluación de la localización de la vena mesentérica superior con respecto a la arteria mesentérica superior y la posición de la tercera porción del duodeno posterior a la arteria mesentérica superior. El ultrasonido permite descartar otras patologías causantes del cuadro de vómito como la estenosis pilórica hipertrófica( ${ }^{(9)}$.

El tratamiento de elección para la malrotación intestinal es el procedimiento de Ladd que consiste en reubicar el duodeno en la gotera derecha del abdomen y el ciego en el cuadrante izquierdo superior con lisis de las bandas sobre el duodeno y apendicetomía, esta última se realiza ya que una apendicitis aguda en el lado izquierdo puede conducir a un mal diagnóstico(6). Estudios han demostrado que tanto el procedimiento vía abierta como el procedimiento via laparoscópica son igualmente viables, seguros y eficaces ${ }^{(10)}$. Sin embargo, se puede observar complicaciones en un $46 \%$ de los pacientes como obstrucción del intestino delgado y síndrome de intestino corto que requiere intervenciones adicionales ${ }^{(4)}$. En cuanto a las membranas duodenales la resección endoscópica está alcanzando una eficacia similar a la intervención quirúrgica abierta con disminución en el número de complicaciones $^{(7)}$.

\section{Conclusión}

La obstrucción duodenal tiene una prevalencia relativamente común en el neonato. Se puede relacionar con malformaciones cardiacas y aneuploidías en recién nacidos. Tiene tanto causas extrínsecas como intrínsecas y es importante tener una sospecha clínica al evidenciar vomito postprandial en un recién nacido, el cual constituye el síntoma principal. Adicionalmente las imágenes diagnósticas son de gran utilidad para su aproximación y manejo oportuno y la resección quirúrgica tanto endoscópica como abierta son el pilar fundamental en el tratamiento.

\section{Referencias}

1. Hernanz-Schulman, M. Duodeonum and Small Bowel: Congenital and Neonatal Abdnormalities. In: B. Coley, ed., Caffey's Pediatric Diagnostic Imaging, 13th ed. Philadelphia, PA: Elsevier; 2019; 953-978.

2. Sinha A., Ahmad M., Kumar B., Kumar, P. Congenital duodenal obstruction due to duodenal web with annular pancreas: A rarity. Archives of International Surgery, 2017; 7(4): 142-144.

3. Bailey P., Tracy T., Connors R., Mooney D., Lewis J., Weber, T. Congenital duodenal obstruction: A 32-year review. Journal of Pediatric Surgery, 1993; 28(1): 92-95.

4. Eksarko P., Nazir S., Kessler E., LeBlanc P., Zeidman, M., Asarian A., Xiao P., Pappas P. Duodenal web associated with malrotation and review of literature. Journal of Surgical Case Reports, 2013; 1(12): 1-3.

5. Jacobs C., Khan F., Apple C.,Larson S. Duodenal web presenting outside the neonatal period concurrently with malrotation and mid-gut volvulus. Journal of Pediatric Surgery Case Reports, 2018; 39: 35-37.

6. Ingoe R., Lange P. The Ladd's Procedure for Correction of Intestinal Malrotation With Volvulus in Children. AORN Journal, 2007; 85(2): 300-308.

7. Beeks A., Gosche J., Giles H., Nowicki, M. Endoscopic Dilation and Partial Resection of a Duodenal Web in an Infant. Journal of Pediatric Gastroenterology and Nutrition, 2009; 48(3): 378-381.

8. Walters M., Robertson R. Gastrointestinal Imaging. In: S. DiPerna and C. Buonomo, ed., Pediatric Radiology: The Requisites, 4th ed. Philadelphia, PA: Elsevier, Inc., 2017; 91-117.

9. Raske Molly E., Dempsey Molly E., Dillman Jonathan R. et al. Vomiting in infants up to 3 months of age. American College of Radiology ACR Appropriateness Criteria, 2014: 1-10.

10. Palanivelu C., Rangarajan M., Shetty A., Jani K. Intestinal malrotation with midgut volvulus presenting as acute Abdomen in children: Value of diagnostic and therapeutic laparoscopy. Journal of Laparoendoscopic \& Advanced Surgical Techniques, 2007; 4(17): 492. 